\title{
Theory for the Change of the Bond Character in Divalent-Metal Clusters
}

\author{
M. E. Garcia, (1) G. M. Pastor, ${ }^{(2)}$ and K. H. Bennemann ${ }^{(1)}$ \\ (1) Institut für Theoretische Physik der Freien Universität Berlin, Arnimallee 14,W-1000 Berlin 33, Germany \\ ${ }^{(2)}$ Institut für Theoretische Physik der Universität zu Köln, Zülpicherstrasse 77, W-5000 Köln 41, Germany
}

(Received 25 March 1991)

\begin{abstract}
To determine the size dependence of the bonding in divalent-metal clusters we use a many-electron Hamiltonian describing the interplay between van der Waals (vdW) and covalent interactions. Using a saddle-point slave-boson method and taking into account the size-dependent screening of charge fluctuations, we obtain for $\mathrm{Hg}_{n}$ a sharp transition from vdW to covalent bonding for increasing $n$. We show also, by solving the model Hamiltonian exactly, that for divalent metals vdW and covalent bonding coexist already in the dimers.
\end{abstract}

PACS numbers: $71.30 .+\mathrm{h}, 36.40 .+\mathrm{d}, 71.10 .+\mathrm{x}$

Divalent-metal atoms are characterized by a closedshell electronic configuration $\left(n s^{2} n p^{0}\right)$, whereas the corresponding solids have $s$ and $p$ bands which overlap and thus give rise to metallic bulk behavior. Therefore, clusters of these elements are expected to exhibit a strong change in their electronic properties as a function of cluster size. This physical picture seems to be confirmed by recent experiments for $\mathrm{Hg}_{n}$ [1-3], which are interpreted as reflecting a size-dependent transition from van der Waals (vdW) to covalent bonding and for larger clusters to metallic bonding [4]. So far, an electronic theory for the size dependence of the bonding and consequently for the transition from van der Waals-like to covalent to metalliclike bonding is lacking. It is the goal of this Letter to present such a theory treating vdW and covalent interactions on the same electronic level.

The dynamics of the valence electrons in divalent-metal clusters is dominated by two distinct interactions: The $\mathrm{vdW}$ interactions (induced dipole-dipole), which involve mainly intra-atomic $s p$ excitations [see inset (b) of Fig. 1], and the covalent interactions, which result from in-

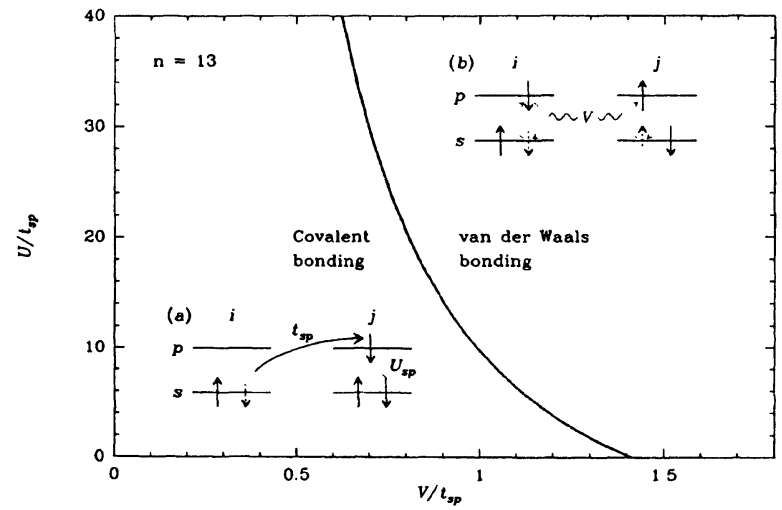

FIG. 1. Phase diagram for $v d W$ and covalent bonding as a function of the Coulomb energy of a charge fluctuation $U$ and the vdW coupling constant $V$ (both scaled by $t_{s p}$ ) for $n=13$ atoms (see Refs. [13] and [14]). In the inset figures the dominant electronic excitations responsible for (a) covalent and (b) $v d W$ interactions are illustrated. teratomic sp transitions and consequently involve charge fluctuations and Coulomb repulsions [see inset (a) of Fig. 1]. Since the same electrons take part in both cohesion processes, there is an interplay between $\mathrm{vdW}$ and covalent interactions which must be taken carefully into account. Consequently, we propose the Hamiltonian [5]

$$
H=H_{\mathrm{vdW}}+H_{\text {cov }} \text {. }
$$

Here vdW interactions involving intra-atomic sp excitations are described by

$$
\begin{aligned}
H_{\mathrm{vdW}}= & \frac{1}{2} \sum_{i \neq j, p \sigma \sigma^{\prime}} V_{i j} c_{i p \sigma}^{\dagger} c_{i s \sigma} c_{j p \sigma^{\prime}}^{\dagger} c_{j s \sigma^{\prime}} \\
& -\frac{1}{2} \sum_{i \neq j, p \sigma \sigma^{\prime}} V_{i j} c_{i p \sigma}^{\dagger} c_{i s \sigma} c_{j s \sigma^{\prime}}^{\dagger} c_{j p \sigma^{\prime}}+\text { H.c. },
\end{aligned}
$$

and covalent interactions due to interatomic transitions involving $s$ and $p$ states are described by

$$
\begin{aligned}
H_{\mathrm{cov}}= & \sum_{i \beta \sigma} \varepsilon_{\beta} c_{i \beta \sigma}^{\dagger} c_{i \beta \sigma}+\sum_{i \neq j, \beta \gamma \sigma} t_{\beta \gamma}^{i j} c_{i \beta \sigma}^{\dagger} c_{j \gamma \sigma} \\
& +\sum_{i, \beta \sigma \neq \gamma \sigma^{\prime}} U_{\beta \gamma} n_{i \beta \sigma} n_{i \gamma \sigma^{\prime}} .
\end{aligned}
$$

The operators $c_{i \beta \sigma}^{\dagger}, c_{i \beta \sigma}$, and $n_{i \beta \sigma}=c_{i \beta \sigma}^{\dagger} c_{i \beta \sigma}$ refer to the creation, annihilation, and occupation-number operators for an electron with spin $\sigma$ at site $i$ and orbital $\beta=s, p$. $V_{i j}$ is the vdW coupling constant between sites $i$ and $j$, which depends on the $s p$ atomic energy gap $\Delta$, the $s p$ contribution to the atomic polarizability $\alpha$, and the interatomic distance $r_{i j}\left(V_{i j} \propto \Delta \alpha / r_{i j}^{3}\right)$ [6]. $H_{\text {cov }}$ is a two-band Hubbard Hamiltonian, where $\varepsilon_{\beta}$ refer to the on-site energies, $t_{\beta \gamma}^{i j}$ to the hopping integrals, and $U_{\beta \gamma}$ to the on-site Coulomb interactions. The Coulomb energy $U$ involved in a local charge fluctuation [e.g., $U=2 U_{s p}-U_{s s}$ from the inset (a) of Fig. 1] actually depends on the cluster size due to the size dependence of screening. For small clusters we expect $U$ to be a sensitive function of the coordination number, as will be discussed below.

An exact solution of the many-body Hamiltonian $H$ is not available at present. A correct treatment of the interplay between vdW and covalent bonding inherent in $H$ requires one to take into account explicitly the different local electronic configurations which contribute to the correlated ground-state wave function. Therefore, we in- 
troduce, in analogy with the slave-boson method of Ref. [7], a set of boson operators which project onto the atomic configurations illustrated in the insets of Fig. 1. The creation (annihilation) operators $s_{i \sigma}^{\dagger}\left(s_{i \sigma}\right), d_{i}^{\dagger}\left(d_{i}\right), m_{i \sigma \bar{\sigma}}^{\dagger}\left(m_{i \sigma \bar{\sigma}}\right)$, and $p_{i \sigma}^{\dagger}\left(p_{i \sigma}\right)$ correspond, respectively, to the configurations $n s^{1}, n s^{2}, n s^{1} n p^{1}$ (with opposite spins), and $n s^{2} n p^{1}$. The physical states in the enlarged Hilbert space (including fermions and bosons) must satisfy

$$
n_{s \sigma}=s_{i \sigma}^{\dagger} s_{i \sigma}+d_{i}^{\dagger} d_{i}+m_{i \sigma \bar{\sigma}}^{\dagger} m_{i \sigma \bar{\sigma}}+\sum_{\sigma^{\prime}} p_{i \sigma^{\prime}}^{\dagger} p_{i \sigma^{\prime}}, \quad n_{p \sigma}=p_{i \sigma}^{\dagger} p_{i \sigma}+m_{i \bar{\sigma} \sigma}^{\dagger} m_{i \bar{\sigma} \sigma},
$$

and the normalization condition

$$
d_{i}^{\dagger} d_{i}+\sum_{\sigma}\left(s_{i \sigma}^{\dagger} s_{i \sigma}+p_{i \sigma}^{\dagger} p_{i \sigma}+m_{i \sigma \bar{\sigma}}^{\dagger} m_{i \sigma \bar{\sigma}}\right)=1 \text {. }
$$

Notice that atomic configurations having no $s$ or more than one $p$ electron have been projected out, since they are not expected to be relevant for the ground state of small neutral $\mathrm{Hg}_{n}$ clusters.

In the new representation the Hamiltonian is given in terms of the fermion and boson operators as

$$
\begin{aligned}
\tilde{H}= & \sum_{i \beta \sigma} \varepsilon_{\beta} c_{i \beta \sigma}^{\dagger} c_{i \beta \sigma}+\frac{1}{2} \sum_{i \neq j, \sigma \sigma^{\prime}} V_{i j} \Omega_{i \sigma}^{\dagger} \Omega_{j \sigma^{\prime}}^{\dagger} c_{i p \sigma}^{\dagger} c_{i s \sigma} c_{j p \sigma^{\prime}}^{\dagger} c_{j s \sigma^{\prime}}-\frac{1}{2} \sum_{i \neq j, \sigma \sigma^{\prime}} V_{i j} \Omega_{i \sigma}^{\dagger} \Omega_{j \sigma^{\prime}} c_{i p \sigma}^{\dagger} c_{i s \sigma} c_{j s \sigma^{\prime}}^{\dagger} c_{j p \sigma^{\prime}}+\text { H.c. } \\
& +\sum_{i \neq j, \beta \gamma \sigma} t_{\beta \gamma}^{i j} Z_{i \beta \sigma}^{\dagger} Z_{j \gamma \sigma} c_{i \beta \sigma}^{\dagger} c_{j \gamma \sigma}+U_{s s} \sum_{i} d_{i}^{\dagger} d_{i}+\left(U_{s s}+2 U_{s p}\right) \sum_{i \sigma} p_{i \sigma}^{\dagger} p_{i \sigma}+U_{s p} \sum_{i \sigma} m_{i \sigma \sigma}^{\dagger} m_{i \sigma \bar{\sigma}}
\end{aligned}
$$

The operator $\Omega_{i \sigma}^{\dagger}\left(\Omega_{i \sigma}\right)$ changes the boson occupations if an intra-atomic $s p$ excitation is created (annihilated) at site $i . \Omega_{i \sigma}^{\dagger}$ is given by

$$
\begin{aligned}
\Omega_{i \sigma}^{\dagger}= & \left(1-s_{i \sigma}^{\dagger} s_{i \sigma}-d_{i}^{\dagger} d_{i}-m_{i \sigma \bar{\sigma}}^{\dagger} m_{i \sigma \bar{\sigma}}-p_{i \bar{\sigma} \overline{i \bar{\sigma}}}^{\dagger}\right)^{-1 / 2} \\
& \times m_{i \bar{\sigma} \sigma}^{\dagger} d_{i}\left(1-m_{i \bar{\sigma} \sigma}^{\dagger} m_{i \bar{\sigma} \bar{\sigma}}\right)^{-1 / 2} .
\end{aligned}
$$

Similarly, $Z_{i \beta \sigma}^{\dagger}\left(Z_{i \beta \sigma}\right)$ changes the boson occupations according to the creation (annihilation) of an electron of spin $\sigma$ at site $i$ and orbital $\beta$. $Z_{i \beta \sigma}$ is the natural generalization for two bands of the operator $\tilde{z}_{i \sigma}$ of Ref. [7] [5,8].

The ground-state properties are evaluated in the saddle-point approximation (SPA) [7]. Here, the boson operators $p_{i \sigma}^{\dagger}, d_{i}^{\dagger}$, etc., are taken as numbers determined by minimizing the ground-state energy [taking into account the constraints (4) and (5)]. Thus, both the hopping elements and the vdW coupling constant become renormalized by the factors

$q_{\beta \gamma \sigma}^{\prime}=\left\langle Z_{\beta \sigma}^{\dagger} Z_{\gamma \sigma}\right\rangle-p^{2}(d+m)^{2} /\left(m^{2}+p^{2}\right)\left(m^{2}+3 p^{2}+d^{2}\right)$

and

$q_{\sigma \sigma^{\prime}}^{V}=\left\langle\Omega_{\sigma}^{\dagger} \Omega_{\sigma}^{\dagger}\right\rangle=d^{2} m^{2} /\left(m^{2}+2 p^{2}\right)\left(m^{2}+4 p^{2}+d^{2}\right)$,

respectively [9]. This reflects in a physically transparent way the interplay between covalent and vdW bonding. The factors $q^{t}$ and $q^{V}$ can be interpreted as order parameters. For example, $q^{t}=0$ means that triple and single occupations vanish, implying localized electrons ( $\mathrm{vdW}$ bonding), whereas $q^{t}=1$ means maximal covalent bonding (delocalized electrons). A transition from localized to delocalized electronic states should be reflected in the cluster-size dependence of $q^{t}$ and $q^{V}$. The competition between itinerancy and vdW interactions can be already seen by noting that $0 \leq q^{t}+q^{V} \leq 1$.

Using Eqs. (4)-(6) in the SPA the ground-state energy
$E_{n}$ of a cluster can be written as

$$
\begin{aligned}
E_{n}= & 2 \sum_{\beta} \int d \varepsilon\left(\varepsilon-\tilde{\varepsilon}_{\beta}\right) \tilde{N}_{\beta}(\varepsilon)+\sum_{i j} \frac{\left(q^{V} V_{i j}\right)^{2}}{\tilde{\varepsilon}_{s}-\tilde{\varepsilon}_{p}}+2 n U p^{2} \\
& +2 n \lambda_{s}^{(2)}\left(d^{2}+3 p^{2}+m^{2}\right)+2 n \lambda_{p}^{(2)}\left(p^{2}+m^{2}\right) \\
& +n \lambda^{(1)}\left(d^{2}+4 p^{2}+2 m^{2}\right)
\end{aligned}
$$

where $\tilde{\varepsilon}_{\beta}=\varepsilon_{\beta}+\lambda_{\beta}^{(2)}$, and $\lambda_{\beta}^{(2)}, \lambda^{(1)}$ are the Lagrange multipliers resulting from the constraints (4) and (5). The first term in Eq. (8) is the kinetic energy, where $\tilde{N}_{s}\left(\tilde{N}_{p}\right)$ refers to the total $s(p)$ density of states calculated using the renormalized hopping elements $\tilde{t}_{\beta \gamma}^{i j}=q^{t} t_{\beta \gamma}^{i j}$ and the shifted on-site energies $\tilde{\varepsilon}_{\beta}$. The second term refers to the vdW energy, which is calculated to second order in $\tilde{V}=q^{V} V$. This is a good approximation, since $\tilde{\varepsilon}_{p}-\tilde{\varepsilon}_{s} \gg \tilde{V}$. The third term is the Coulomb energy due to interatomic charge fluctuations [see inset (a) of Fig. 1]. For simplicity we assume $U_{\beta \gamma}=U$ and we drop the site dependences (i.e., $t_{\beta \gamma}^{i j}=t_{\beta \gamma}$ and $V_{i j}=V$ ) by restricting the sums to nearest neighbors. The saddle-point (SP) values of the boson fields and renormalization factors $q^{t}$ and $q^{V}$ have been obtained by minimizing $E_{n}\left(s^{2}, m^{2}, p^{2}, d^{2}\right)$ for clusters with $n \leq 43$ atoms and different values of the interaction parameters $U$ and $V[10]$.

For $V=0$ we obtain, for all cluster sizes, a single minimum in $E_{n}$ having $q^{t}=1$ and $q^{V}=0$. This corresponds to the SP solution of the two-band Hubbard model with two electrons per site. Here, $p^{2}=s^{2} \neq 0\left[p^{2}\right.$ $-t_{s p}^{2} /(\Delta+U)^{2}$ for $\left.U \rightarrow \infty\right]$, whereas $m^{2}$ is negligible $\left(p^{2} \gg m^{2}\right)$. The binding energy $E_{n}\left(q^{t}-1\right)$ decreases for increasing $U$, and particularly $E_{n} \sim t_{s p}^{2} /(\Delta+U) \rightarrow 0$ for $U \rightarrow \infty$. The binding is, as expected, exclusively due to interatomic charge fluctuations.

New features appear if vdW interactions are included. For $V \neq 0$, in addition to the covalent minimum a second 
SP is found in $E_{n}$ having $q^{V}-1$ and $m^{2} \sim(V / \Delta)^{2} \neq 0$, while $q^{t}=0$ and $p^{2} \ll m^{2}$. At this SP the excitations illustrated in the inset (b) of Fig. 1 dominate and the energy is independent of $U\left(E_{n} \sim V^{2} / \Delta\right)$. The bonding is of pure vdW character. The SP which is actually relevant for the cluster ground state is the one with the lowest energy. The resulting phase diagram for the bond character as a function of $U$ and $V$ is shown in Fig. 1 for a cluster with $n=13$ atoms [11]. A similar phase diagram is obtained for other cluster sizes $(n \leq 43)$ provided that the $s$ and $p$ bands do not overlap. The importance of treating $\mathrm{vdW}$ and covalent interactions on the same microscopic level is evident. Furthermore, from Fig. 1 one may conclude already quite generally that a transition from vdW to covalent bonding can occur for increasing $n$, since the energy $U$ involved in a local charge fluctuation should decrease with increasing $\boldsymbol{n}$ due to screening.

To demonstrate that our theory is able to explain quantitatively the change in the bond character of $\mathrm{Hg}_{n}$ clusters as a function of cluster size, we estimate the interaction parameters $V$ and $U$ as follows. The vdW coupling constant [5] $V=c \alpha \Delta / r_{0}^{3}=0.55 \mathrm{eV}$ is obtained from the atomic polarizability per valence electron $\alpha=2.85 \AA^{3}$, the average between the dimer and the bulk nearest-neighbor distances $r_{0}=3.33 \AA$, and the constant $c \simeq 1.25$, which was determined by fitting to the binding energy of raregas dimers. The Coulomb energy $U$ involved in an interatomic charge fluctuation is given by $U=U_{0}-U_{1}-\Delta U$, where $U_{0}=2 U_{s p}-U_{s s}$ stands for the increase of the intra-atomic Coulomb repulsion, and $U_{1} \simeq\left(U_{0}^{-1}+r_{0} /\right.$ $\left.e^{2}\right)^{-1}$ for the remaining electron-hole interaction [see inset (a) of Fig. 1]. $\Delta U$ takes into account the screening of interatomic charge fluctuations due to the polarization of the atoms surrounding the electron-hole pair. Using

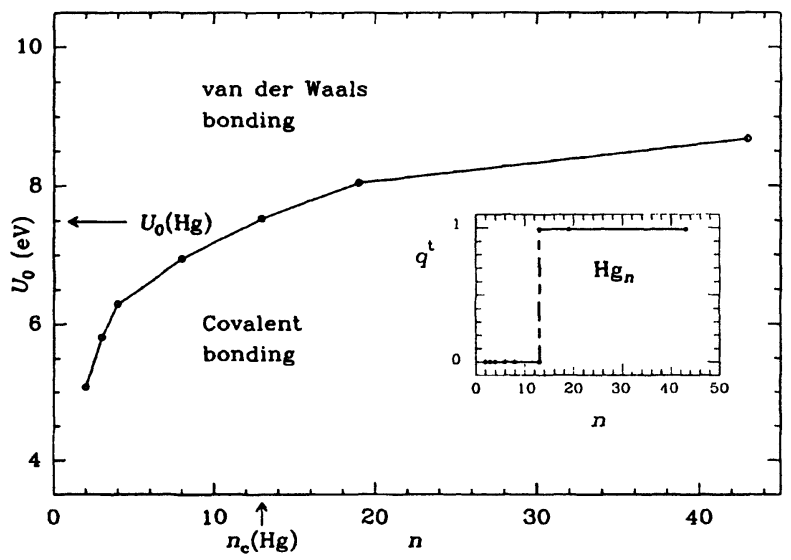

FIG. 2. Phase diagram for vdW and covalent bonding as a function of the intra-atomic Coulomb repulsion $U_{0}$ and $n$. Estimating $U_{0}(\mathrm{Hg})=7.5 \mathrm{eV}$, one obtains $n_{c}(\mathrm{Hg})=13$ atoms. Inset: The resulting behavior of $q^{\prime}$ for $\mathrm{Hg}_{n}$. second-order perturbation theory one obtains for the screening resulting from the charge-dipole interaction

$\Delta U=\frac{a e^{2}}{r_{0}^{4}}\left\langle\sum_{k}^{\prime}\left[\left(\frac{r_{0}}{r_{k i}}\right)^{4}+\left(\frac{r_{0}}{r_{k j}}\right)^{4}-2 r_{0}^{4} \frac{\mathbf{r}_{k i} \cdot \mathbf{r}_{k j}}{r_{k i}^{3} r_{k j}^{3}}+1\right]\right\rangle_{i j}$,

where $\langle\cdots\rangle_{i j}$ indicates cluster average through all nearest neighbors $i j$ at which the charge fluctuation occurs. As physically expected, $\Delta U$ yields a reduction of $U$ with increasing $n$ due to the increase of the coordination number [12].

In Fig. 2 results are given for the bond character of $\mathrm{Hg}_{n}$ clusters as a function of $U_{0}$ and $n$ [11]. The phase boundary is determined using Eqs. (8) and (9) as the value of $U_{0}$ for which $n$ is equal to the critical cluster size $n_{c}$ at which the transition from vdW to covalent bonding occurs. An approximated realistic value of $U_{0}$ for $\mathrm{Hg}$ is indicated [13]. According to this the transition occurs in $\mathrm{Hg}_{n}$ at $n_{c}=10-20$ atoms. Although the results depend implicitly on the other parameters $\left(V, \Delta, t_{s p}\right)$, Fig. 2 can be interpreted as a phase diagram for the bond character. Notice that the existence of a transition is restricted to a certain range of values for $U_{0}$. For instance, if $U_{0}<5$ $\mathrm{eV}$, the system would be covalent already for $n=2$. For $U_{0}>9 \mathrm{eV} \mathrm{vdW}$ behavior results for all cluster sizes, as we obtain for rare-gas clusters. If $\Delta U$ is neglected the range for the occurrence of the transition is reduced. In the inset of Fig. 2 the size dependence of the hopping renormalization factor $Q^{t}$ of $\mathrm{Hg}_{n}$ is shown. For $n \leq 13$, $q^{t}=0$ implies that the electrons are localized. Only vdW bonding is present $\left(q^{V}=1\right)$ and the binding energy per bond is roughly independent of $n$. At $n=13$ there is a crossing of the SP solutions and the character of the

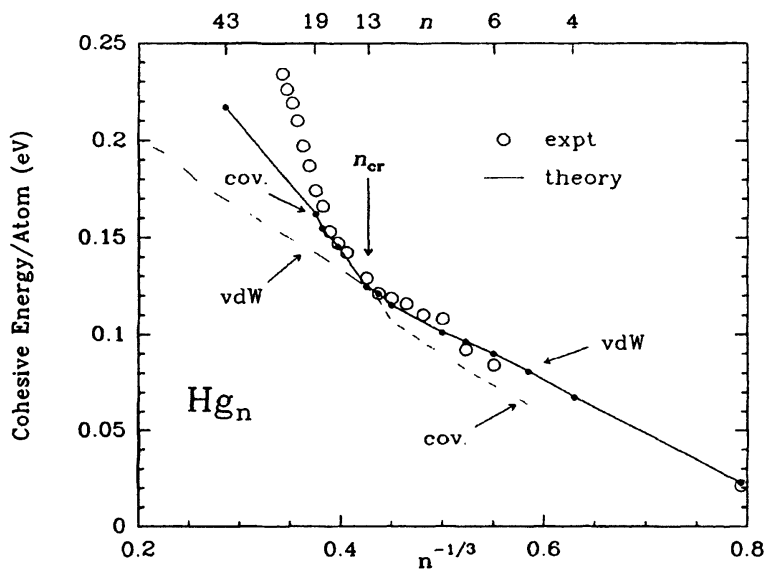

FIG. 3. Size dependence of the cohesive energy per atom. Open circles refer to the experimental values (Ref. [3]), and solid circles to the theory (this work). The dashed lines indicate results for the unstable covalent state $(n<13)$ and the unstable vdW state $(n>13)$. 
bonding changes. For $n>13$, we obtain $q^{\prime}=1$ which physically means that the valence electrons delocalize to form covalent bonds, or for larger clusters metallic bonds, and $\mathrm{vdW}$ bonding is suppressed $\left(q^{V}=0\right)$. Now the binding energy per bond increases with $n$. This change in the bond character is also reflected in the size dependence of the cohesive energy $E_{\text {coh }}(n)$ [14]. As shown in Fig. 3, a change of the slope occurs at $n \approx 13$, which results from the crossing of the vdW and covalent SP. Quantitatively our results are in good agreement with experiment [3]. The discrepancies for $n>19$ are probably due to the approximation used for the screening [Eq. (9)], which might tend to overestimate the repulsive Coulomb energy of charge fluctuations in clusters with dominant covalent character. The calculated suppression of band formation $\left(q^{\prime}=0\right)$ for $n<n_{c}$ and the change to delocalized valence electrons $\left(q^{t}=1\right)$ for $n>n_{c}$ is also in agreement with the following observations on $\mathrm{Hg}_{n}$ : For $n \lesssim 13$ the $5 d \rightarrow 6 p$ autoionization lines are symmetric (atomiclike) and the line widths are approximately independent of $n$, while for $n \gtrsim 20$ they broaden asymmetrically indicating $6 p$ band formation [1]. For $n \lesssim 13$ the size dependence of the ionization energy $I_{n}$ is very similar to that of vdW clusters, while for $n \gtrsim 13 I_{n}$ decreases more rapidly indicating $6 s$ band broadening [2].

In order to study the interplay between vdW and covalent bonding beyond the SPA we diagonalize exactly the Hamiltonian $H$ [Eqs. (1)-(3)] for $n=2$. For $V \neq 0$, $t_{\beta \gamma} \neq 0$, and finite $U$, we obtain coexistence of vdW and covalent bonding. For instance, we estimate that the vdW contribution to the cohesive energy is more dominant for $\mathrm{Hg}_{2}$ and $\mathrm{Mg}_{2}(\sim 80 \%)$ than for $\mathrm{Ca}_{2}(\sim 60 \%)$ [5]. The origin of the coexistence can be understood by noting that the electronic configurations involved in $\mathrm{vdW}$ and covalent bonding are interconnected by the terms $t_{s p} c_{i p \sigma}^{\dagger} c_{j s \sigma}$ in $H$, for example.

Thus, quantum fluctuations between the vdW and covalent SP are present, which cause the actual ground state to be a combination of both vdW and covalent contributions.

Consequently, the transition from vdW to covalent bonding as a function of cluster size should be somewhat smoother than what the SPA calculations yield (Fig. 2).

The exact results $(n-2)$ agree with the SP slave-boson calculations. For instance, the boundary between vdW and covalent behavior as a function of $V / t_{s p}$ and $U / t_{s p}$ compares well with the SPA results given in Fig. 1. The physical picture supplied by the slave-boson calculations seems to account for the essential features of the character of the bonding in divalent-metal clusters.

Summarizing, we have calculated, for the first time, the transition from vdW to covalent bonding in $\mathrm{Hg}_{n}$ clusters. The importance of treating $\mathrm{vdW}$ and covalent interactions on the same electronic level has been demonstrated. Our theory should serve as a basis for calculating other electronic properties of divalent-metal clusters.

Helpful discussions with P. Stampfli, W. Zhang, and B. Mühlschlegel are gratefully acknowledged. This work has been supported by the Deutsche Forschungsgemeinshaft through Sonderforschungsbereich No. 337 and No. 341 .

[1] C. Bréchignac, M. Broyer, Ph. Cahuzac, G. Delacretaz, P. Labastie, J. P. Wolf, and L. Wöste, Phys. Rev. Lett. 60, 275 (1988).

[2] K. Rademann, B. Kaiser, U. Even, and F. Hensel, Phys. Rev. Lett. 59, 2319 (1987).

[3] H. Haberland, H. Kornemeier, H. Langosch, M. Oschwald, and G. Tanner, J. Chem. Soc. Faraday Trans. 86, 2473 (1990).

[4] G. M. Pastor, P. Stampfli, and K. H. Bennemann, Phys. Scr. 38, 623 (1988); Europhys. Lett. 7, 419 (1988).

[5] Further details will be published elsewhere.

[6] In fact, the coupling constants for the first and second terms in $H_{v d w}$ should be different due to Coulomb interactions. However, this has no consequences for the ground-state properties discussed here.

[7] G. Kotliar and A. E. Ruckenstein, Phys. Rev. Lett. 57, 1362 (1986).

[8] In the SPA, $Z_{i \sigma}$ and $\boldsymbol{\Omega}_{i \sigma}$ yield for all band fillings the exact result both for the noninteracting limit $(U=0, V=0)$ and the pure vdW limit $(t=0)$. See also Ref. [7].

[9] For simplicity, the site-dependent boson fields have been replaced by their respective cluster averages $s^{2}, d^{2}$, etc.

[10] Results shown in Figs. 1-3 were obtained by using the close-packed structures given in Ref. [4] (i.e., for $n \geq 13$ fcc-like). Our results and conclusions were checked to be insensitive to the details of the cluster structure.

[11] We use $\Delta=5.8 \mathrm{eV}, t_{s p}=0.5 \mathrm{eV}, t_{s s}=0.44 \mathrm{eV}$, and $t_{p p}=0.73 \mathrm{eV}$, which correspond approximately to $\mathrm{Hg}$ (Ref. [4]).

[12] A more accurate treatment of the size-dependent screening is, of course, desirable. However, such improvements would not change our conclusions.

[13] C. Moore, Atomic Energy Levels, National Bureau of Standards Circular No. 467 (U.S. GPO, Washington, DC, 1958).

[14] $E_{\text {coh }}(n)=-\left[E_{n}+(z / 2) E_{B M}\right] / n$. Here $z$ is the average coordination number and $E_{B M}$ is a constant core-core repulsive energy, which was fitted to the experimental $E_{\text {con }}(2)$ and has no influence on the change of the slope in Fig. 3. 